\title{
Joint effect of lattice interaction and potential fluctuation in colossal magnetoresistive manganites
}

\author{
R. Y. Gu \\ Texas Center for Superconductivity, University of Houston, Houston, TX 77204 \\ and Ames Labortory, Iowa State University, Ames, IA 50011
}

(December 20, 2018)

\begin{abstract}
Taking into account both the Jahn-Teller lattice distortion and the on-site electronic potential fluctuations in the orbital-degenerated double-exchange model, in which both the core-spin and the lattice distortion are treated classically, we investigate theoretically the metal-insulator transition (MIT) in manganites by considering the electronic localization effect. An inverse matrix method is developed for calculation in which we use the inverse of the transfer matrix to obtain the localization length. We find that within reasonable range of parameters, both the lattice effect and the potential fluctuation are responsible to the occurrence of the MIT. The role of the orbital configuration is also discussed.
\end{abstract}

PACS numbers: 71.30.+h, 71.38.-k, 72.15.Gd 


\section{INTRODUCTION}

Colossal magnetoresistance (CMR), an effect discovered in mixed-valence manganites $\mathrm{R}_{1-x} \mathrm{~A}_{x} \mathrm{MnO}_{3}$ (where $\mathrm{R}$ and $\mathrm{A}$ are rare and alkaline-earth ions, respectively) have attracted much attention [1-5]. In the hole doping range $0.17<x<0.4$, such an effect occurs in the vicinity of the metal-insulator transition (MIT) temperature $T_{M I}$ at which the resistivity of the system varies drastically. It is generally accepted that the anomalous transport phenomena in these manganese systems are closely related to their magnetic properties, in particular the paramagnetic (PM) to ferromagnetic (FM) phase transition upon cooling, as in most experimental measurements, $T_{M I}$ and the magnetic Curie temperature $T_{c}$ are very close to each other.

The double exchange (DE) mechanism [6] is believed to play a major role in the magnetic transition, in which the carriers are ferromagnetically coupled to the Mn core spin due to a strong Hund's coupling, resulting in the hopping amplitude depending on the relative Mn spin orientation. On the other hand, the mechanism responsible for the MIT has not yet been very clear [7]. It was found that in single orbital calculations the random hopping from the DE mechanism alone is not enough to drive the MIT [8-10]. Many further theoretical efforts have been made to understand the MIT. By taking into account the potential fluctuations experienced by carriers due to the random distribution of $R^{3+}$ and $A^{2+}$, Sheng et al. [8] proposed that the electrons are localized above $T_{c}$ due to strong on-site and spin disorder, while at low temperature the alignment of the spins reduce the spin disorder and the electrons are delocalized. In their calculation, however, the strength of the on-site disorder required for the localization, $W \geq 12 t$ ( $W$ is the range of the random on-site energy distribution, and $t$ is the one-orbital hopping amplitude), is apparently overestimated, as the reasonable magnitude of $t$ is roughly between 0.2 and $0.5 \mathrm{eV}$ [11], while the potential fluctuations would amount to $1.7 \mathrm{eV}$ if unscreened, and be further reduced when screening is accounted [4]. Millis et al. [12] used the dynamical mean-field method to study the coupling of the carriers to local Jahn-Teller (JT) distortions and to the Mn core spins, but an MIT was found 
only at half filling $(x=0)$. In recent years, there are also much interest on phase separation scenario [13-15], in which the metallic and insulating regions coexist in the system and the transport is a percolation problem, near the percolation critical point, a small change of the fraction of the metallic regions can induce the MIT. But why this fraction change occurs with the variation of temperature still needs an explanation. Very recently, Vergés et al. [16] proposed a lattice-spin mechanism to explain the MIT, by using Monto Carlo simulation in a $6^{3}$ lattice for a single-orbital DE model coupled with phonons, they found that at $x=0.08$, in a narrow region of the coupling parameter, an MIT coincidence with the FM to PM transition upon heating.

Based on previous experimental and theoretical studies, it appears that the following scattering mechanisms are important to the transport in manganites: (i) the strong DE interaction between carriers and the localized spin, which is usually regarded as the basic mechanism in manganites, (ii) the potential fluctuations experienced by the electrons due to the random $R^{3+}$ and $A^{2+}$ ion distribution, according to Ref.[4], this fluctuations might be comparable to the electron band-width, and (iii) the JT distortion which was revealed in many experimental measurements. Besides the basic DE interaction (i), in Refs.[8] and [16], (ii) and (iii) were considered, respectively. Yet both theories have difficulties in their explanation of MIT (overestimated W in the former and not at the right doping in the latter). Furthermore, in recent years it was also realized that in manganites the twofold $e_{g}$ orbital degeneracy and the unique Slater-Koster form of electronic hopping [17] are important to many aspects of the system [18]. In many theoretical treatments, such as in Refs[8] and [16], to simplify the calculation a single orbital model was adopted, as it was believed that the JT distortion would split the $e_{g}$ degeneracy and reduce the problem to an effective single orbital one. However, whether the energy splitting $\Delta$ is large enough to ignore the degeneracy is questionable, in Ref.[16] the electron-lattice coupling responsible to the desired MIT is around $\lambda=1.45$, for such a strength of JT coupling, the average of the corresponding $\Delta$ is about $4(1-\mathrm{x}) \mathrm{t}$, considerably smaller than the width of the energy band $12 t$. On the other hand, even in the $\Delta \rightarrow \infty$ limit where at each site there is only one orbital valid, it 
is still different from the usual single orbital model due to the anistropy of the hopping, for example, in this large $\Delta$ limit if the lower-energy orbit is $d_{x^{2}-y^{2}}$, then along the z-direction there is no electronic hopping, very different from the isotropic hopping in the usual single orbital model.

Because of the problems in previous MIT theories raised above, it is desirable to investigate the MIT of manganites by including all the three scattering mechanisms as well as the orbital degeneracy. Up to now no such theoretical study has been made yet, and we will make such an effort in this work. Starting from an effective degenerated DE model with electron-lattice interaction and potential fluctuation included, we investigate the MIT by considering the electronic localization effect. An inverse matrix method suitable for the present specific Hamiltonian is developed for our calculation. We find that while similar to the single orbital model, in the degenerated orbital case the spin disorder alone is not enough to localize the carriers either, the introduction of the electron-lattice interaction and potential fluctuation, within reasonable regions of parameters, can induce the MIT. We also

discuss the role of orbital disorder in the MIT. It is shown that in the presence of strong electron-lattice interaction, electrons become more difficult to be localized in the orbital disordered state than in the orbital ordered state due to the split of orbital degeneracy and the anistropic electronic hopping in the latter.

\section{MODEL AND METHOD OF CALCULATION}

We consider an effective DE Hamiltonian by taking into account the strong on-site Hund's coupling between the itinant $e_{g}$ electrons and the Mn core spins, together with the coupling between electrons and lattice distortion,

$$
H_{e}=-\sum_{i j} f_{i j} c_{i}^{\dagger} \hat{t}_{i j} c_{j}+\sum_{i} \epsilon_{i} c_{i}^{\dagger} c_{i}-g \sum_{i} Q_{i} c_{i}^{\dagger}\left(\sin \phi_{i} \tau_{x}+\cos \phi_{i} \tau_{z}\right) c_{i}+\frac{1}{2} \sum_{i} Q_{i}^{2}
$$

where $c_{i}^{\dagger}=\left(c_{i+}^{\dagger}, c_{i-}^{\dagger}\right)$ with + and - representing orbital states $d_{3 z^{2}-r^{2}}$ and $d_{x^{2}-y^{2}}$, respectively. The first term of Eq. (1) is the effective DE Hamiltonian in which $f_{i j}=\cos \left(\theta_{i} / 2\right) \cos \left(\theta_{j} / 2\right)+$ 
$\sin \left(\theta_{i} / 2\right) \sin \left(\theta_{j} / 2\right) e^{-i\left(\varphi_{i}-\varphi_{j}\right)}$ with $\left(\theta_{i}, \varphi_{i}\right)$ being the polar angles characterizing the orientation of the localized spin on site $i, \hat{t}_{i j}=\hat{t}^{\alpha}$ are $2 \times 2$ hopping matrix in orbital space, with $\alpha(=x, y, z)$ being the directions of the bond (ij) between neighboring Mn sites, and $\hat{t}_{++}^{x, y}=$ $t / 4, \hat{t}_{+-}^{x, y}=\mp \sqrt{3} t / 4, \hat{t}_{--}^{x, y}=3 t / 4, \hat{t}_{++}^{z}=t, \hat{t}_{+-}^{z}=t_{--}^{z}=0$, and $\hat{t}_{-+}^{\alpha}=\hat{t}_{+-}^{\alpha}$ [13]. The second term represents the potential fluctuations experienced by $e_{g}$ electrons due to the randomness of the $R^{3+}$ and $A^{2+}$ ion cores, for simplity we assume that $\epsilon_{i}$ is uniformly distributed within the range $[-\mathrm{W} / 2, \mathrm{~W} / 2]$ [8]. The last two terms are related to the Jahn-Teller distortion, in which $\tau_{x}, \tau_{z}$ are Pauli matrices and $Q_{i} \sin \phi_{i}, Q_{i} \cos \phi_{i}$ are the two Jahn-Teller modes [12]. Like in many other works, in Eq.(1) we have used the classical limit for both the core spins and the Jahn-Teller distortions. In principle, the quantum lattice and spin fluctations could change some of the results. In the case of large core spin $S=3 / 2$ and static lattice distortion, as is considered in this work, one expects that the quantum effect will not change the quanlitative conclusion of the classical approach.

In the following we will calculate the electronic localization length of Hamiltonian (1) in a long bar. Without losing generality we set the bar along the $\mathrm{z}$ direction, and the width of each slice of the bar is $M$. Usually the localization length $\lambda_{M}$ is obtained through the transfer matrix method [19], which is based on the recursion relationship of successive slices, $A_{n+1}=T_{n}^{-1}\left[\left(E-H_{n}\right) A_{n}-T_{n-1} A_{n-1}\right]$, where $A_{n}$ is the wave function amplitude in the $n t h$ slice, $E$ is the energy of electron, and $H_{n}$ is the $2 M^{2} \times 2 M^{2}$ matrix of the electronic Hamiltonian inside the slice, and $T_{n}$ is a $2 M^{2} \times 2 M^{2}$ diagonal matrix with elements being the electronic hopping amplitudes between neighboring sites in the $n t h$ and $n+1 t h$ slices. The size of the corresponding transfer matrix is $4 M^{2} \times 4 M^{2}$. For the present specific Hamiltonian, this method can not be directly applied as $T_{n}^{-1}$ does not exist due to the lack of hopping between neighboring $d_{x^{2}-y^{2}}$ orbitals along the $\mathrm{z}$ direction. The propagation of $d_{x^{2}-y^{2}}$ in the $\mathrm{z}$ direction is through its mixing with the $d_{3 z^{2}-r^{2}}$ in the transverse directions. As a result, it is adequate to use the recursion equation of the latter to calculate the localization. By eliminating the amplitude of $d_{x^{2}-y^{2}}$ orbitals, the equation reduces to 


$$
B_{n+1}=T_{n}^{\prime-1}\left[\left(E-H_{n}^{++}-U_{n}\right) B_{n}-T_{n-1}^{\prime} B_{n-1}\right]
$$

where $B_{n}$ is the amplitude of $d_{3 z^{2}-r^{2}}$ orbital, $T_{n}^{\prime}$ is the reduced $M^{2} \times M^{2}$ diagonal matrix whose $\nu$ th diagonal elements is the effective hopping $f_{i j}$ between site $\nu\left(\nu=1,2 \ldots, M^{2}\right.$ denote the sites within the slice) in the $n$th slice and its neighboring in the $n+1$ th slice. $H_{n}^{\gamma \gamma^{\prime}}\left(\gamma, \gamma^{\prime}=+,-\right)$ is the $M^{2} \times M^{2}$ submatrix of $H_{n}$ in subspace spanned by basis $\left\{c_{\nu \gamma}^{\dagger}\right\}$ and $\left\{c_{\nu \gamma^{\prime}}\right\}, U_{n}=H_{n}^{+-}\left(E-H_{n}^{--}\right)^{-1} H_{n}^{-+}$is due to the mixing of the $d_{3 z^{2}-r^{2}}$ and $d_{x^{2}-y^{2}}$ orbitals inside the slice.

The $2 M^{2} \times 2 M^{2}$ transfer matrix of each slice can be obtained from Eq.(2). The localization length corresponds to the smallest positive Lyapunov exponent of the product of the transfer matrices, which comes from, if we treat $B_{n}$ as a matrix, the behavior of its smallest eigenvalue in the limit of $n \rightarrow \infty$. In this method, since in a direct computation of the product matrix the information associated with the smallest positive Lyapunov exponent will be lost when the ratio of the contribution from the smallest positive Lyapunov exponent to that of the largest Lyapunov exponent becomes comparable with the machine accuracy, one has to orthonormalize the product matrix regularly [19]. Instead of this method, here we develop an inverse matrix method in which there is no need to do the orthonormalization. Since the smallest eigenvalue of $B_{n}$ corresponds to the largest eigenvalue of $B_{n}^{-1}$, we seek the recursion formula of $B_{n}^{-1}$. From Eq.(2), it is given by

$$
B_{n+1}^{-1}=B_{n}^{-1} p_{n+1}
$$

where the matrix $p_{n+1}$ can be directly obtained from recursion $p_{n+1}=\left[E-H_{n}^{++}-U_{n}-\right.$ $\left.T_{n-1}^{\prime} p_{n}\right]^{-1} T_{n}^{\prime}$. The largest eigenvalue of $B_{n}^{-1}$ is related to the localization length and it increases exponently with $n$, so $B_{n}^{-1}$ needs to be regularly (but not necessarily at each $n$ ) renormalized by divided by $b_{n}=\sqrt{\sum_{i j}\left|\left(B_{n}^{-1}\right)_{i j}\right|^{2}}$. For a bar of length $L$, the localization length is given by $\lambda=L /\left|\sum_{n}^{\prime} \log \left(b_{n}\right)\right|$, in which the sum is over all $\mathrm{n}$ where $B_{n}^{-1}$ are renormalized. The result of $\lambda$ is not sensitive to the initial conditions so that $p_{1}$ and $B_{1}$ can be simply set as the unit matrix. 
The present inverse matrix method has been compared with the transfer matrix method. For the usual localization problems such as those in Ref.[19], both methods give the same results within accuracy. For the present Hamiltonian Eq.(1), we find that the former is more efficient due to the smaller matrix size and no need to orthonormalize in the calculation (the disadvantage of having to inverse the matrix becomes less serious since in the present problem the latter also needs to perform matrix inversion in $U_{n}$ ). Moreover, it is found that the result of the latter lacks stability, for example, the results can be totally different by using single

or double precision calculations, this deficiency may come from $T_{n}^{\prime-1}$ for very small effective hopping. On the other hand, there is no such problem in the former method. The difference in stability between these two methods is the main reason that we choose the inverse matrix method in our calculation.

\section{RESULTS AND DISCUSSION}

\section{A. Pure DE effect}

Now let us first see the case of a pure DE model $(g=W=0)$ with orbital degeneracy. In this case, for a perfect FM phase all electronic states are extended, with the energy band from $-3 t$ to $3 t$. For hole-doped manganites $\mathrm{R}_{1-x} \mathrm{~A}_{x} \mathrm{MnO}_{3}(0 \leq x \leq 1)$, the Fermi energy $\varepsilon_{F}$ is at the bottom of the band near $x=1$, increases with the decreasing of $x$, and reaches the center of the band at $x=0$. With the increase of the randomness of spin configuration, the effective electronic hopping and energy band width decreases. At the PM phase, if we treat $\cos \theta_{i}$ and $\varphi_{i}$ as independent variables that are uniformly distributed in regions $[-1,1]$ and $[-\pi, \pi]$, then the band bottom is at around $-2.5 t$. Under this treatment, Fig. 1 shows the calculated rescaled localization length as a function of energy $\mathrm{E}$ with different width $\mathrm{M}$ of the bar. The length of the bar is taken to be $L=10^{5}$, which is enough for our calculation. All curves are crossed at a fixed point $E_{c} \approx-2.0 t$, which corresponds to the Fermi energy at doping $x_{c} \approx 0.85$. The localization length in the thermodynamical limit $M \rightarrow \infty$ can be 
obtained through the one-parameter scaling theory, in which the rescaled localization length $\lambda_{M} / M$ can be fitted in terms of a universal function $\lambda_{M}(E) / M=f(M / \xi(E))[19]$, and the scaling parameter $\xi$ has two branches (inset of Fig. 1). For $E<E_{c}, \lambda_{M}(E) / M$ decreases toward zero and $\xi$ is the localization length $\lambda_{\infty}(E)$ for an infinite system, so electrons are localized and have no contribution to the conductivity. The electron state becomes extended when $E>E_{c}$, where $\lambda_{M} / M$ increases with $M$ and $\lambda_{\infty}$ is infinite. The present calculation indicates that in a pure DE model with orbital degeneracy, at doping $x<0.85$ in the PM state the DE spin disorder is not enough to localize the carriers on the Fermi surface and the system is metallic. This conclusion is not consistent with the experimental results, where the MIT usually occurs in the range $0.17<x<0.4$.

\section{B. Joint effect}

Next we take the other two scattering mechanisms into account. At each site the JT distortion is described by two quantities $Q_{i}$ and $\phi_{i}$. For the amplitudes of the distortion $Q_{i}$, by minimizing the energy their average can be obtained as $\left\langle Q_{i}\right\rangle=(1-x) g$. To simplify our study in the following calculations we will replace all $Q_{i}$ with $\left\langle Q_{i}\right\rangle$. For the angle $\phi_{i}$, it is closely related to the orbital polarization on site $i$, and since the configuration of orbitals at different sites are disordered at hole concentration $0.17<x<0.4$ where the FM metallic to PM insulator transition occurs, we put $\phi_{i}$ as random variables with uniform distribution between $-\pi$ and $\pi$. Fig. 2 shows the calculated phase diagram for the PM and perfect FM magnetic background at $x=0.2$ in the $g-W$ plane, the energy is taken to be the Fermi energy at each $g$ and $W$. Here we are interested in the region between the two MIT lines $L_{\mathrm{PM}}$ and $L_{\mathrm{FM}}$, which is an insulator in the PM and a metal in the FM case, respectively, so that an MIT will occur in this region when the magnetic configuration is changed from FM to PM. From Fig. 2, at $g=1.6$, to obtain the MIT, the required strength of the onsite disorder is $W \approx 3.5 t$, much smaller than that in Ref.[8] (where $W \geq 12 t$ ), and is a

reasonable magnitude according to the estimation in Ref.[4]. Fig. 2 is the main result of 
this paper, based on it we propose that the FM metallic to PM insulator transition is due to the joint effect of the DE disorder, the JT distortion and the on-site potential fluctuation. It is worth mentioning that in the present calculation we have neglected the fluctuation of the lattice distortion amplitude $Q_{i}$, which in principle depends on the electron occupation state on each site and has a thermal fluctuation around $\left\langle Q_{i}\right\rangle$. Since the on-site energy due to the lattice distortion at each site is proportional to $Q_{i}$, this fluctuation will introduce a potential randomness in addition to that from $W$, so if considered it may further reduce the magnitude of $W$ required for localization.

\section{Effect of orbital configuration}

Since it appears that the on-site potential of orbitals $d_{3 z^{2}-r^{2}}$ and $d_{x^{2}-y^{2}}$ (being $-g Q_{i} \cos \phi_{i}$ and $-g Q_{i} \sin \phi_{i}$, respectively) is more random in the orbital disordered case than in the ordered case and thus the diagonal disorder is stronger in the former, one might expect that electrons are easier to be localized in the orbital disordered state than in the orbital ordered state. To check this point we also perform calculations in the orbital ordered configurations and compare them with that in the orbital disordered case. In Fig. 3 the rescaled localization length in both the orbital disordered and C-type antiferromagnetic (AF) orbital ordered cases are shown, in the latter case $\phi_{i}$ is taken to be $\pm 2 / 3 \pi$ in the two sublattices, corresponding to the $d_{3 x^{2}-r^{2}} / d_{3 y^{2}-r^{2}}$ type order. It is found that contrary to this expection, electrons in the orbital ordered case electrons are actually easier to be localized. Similar results are also obtained for the A-type AF, G-type AF and FM orbital ordered configurations. This finding is consistent with the occurence of the orbital ordered insulator and orbital disordered metal observed in experiments [20]. In fact, for each $\phi_{i}$, one can perform a local unitary transformation $U_{i}$ to transform the orbital state basis from $|+\rangle,|-\rangle$ to $\left|+^{\prime}\right\rangle_{i}=$ $\cos \left(\phi_{i} / 2\right)|+\rangle+\sin \left(\phi_{i} / 2\right)|-\rangle$ and $\left|-^{\prime}\right\rangle_{i}=-\sin \left(\phi_{i} / 2\right)|+\rangle+\cos \left(\phi_{i} / 2\right)|-\rangle$. The electron-lattice interaction becomes diagonal under this transformation, with the on-site energy being $-g Q_{i}$ for state $\left|+^{\prime}\right\rangle_{i}$ and $g Q_{i}$ for $\left|-^{\prime}\right\rangle_{i}$, respectively, which are independent of the orbital polar angle 
$\phi_{i}$. At the same time the hopping term in Eq.(1) is transformed to $f_{i j} U_{i}^{\dagger} \hat{t}_{i j} U_{j}$, so orbital disorder actually does not increase the randomness of the on-site potential fluctuation, it only affects that of the electronic hopping, which is already random in the PM phase due to the spin disorder. To our understanding, the localization effect of the electron-lattice interaction comes from its splitting the energy degeneracy. In the case of energy degeneracy, both orbital states $\left|+^{\prime}\right\rangle_{i}$ and $\left|-^{\prime}\right\rangle_{i}$ are important to electronic transport and electron at a site can hop to either the $\left|+^{\prime}\right\rangle$ or the $\left|-^{\prime}\right\rangle$ orbital state on its neighboring site. The electronlattice interaction introduces an energy gap $\Delta$ between these two states. With the increase of $\Delta$, it becomes more difficult for an electron at $\left|+^{\prime}\right\rangle$ state to move to the neighboring $\left|-^{\prime}\right\rangle$ state due to their energy difference, so that electrons are easier to be localized in this case. To understand that the localization effect is even stronger in the orbital ordered case, one may note that at large $\Delta$, electron hopping occurs mainly between neighboring $\left|+^{\prime}\right\rangle$ states, whose hopping amplitude is proportional to $\cos \left[\left(\phi_{i}-\phi_{\alpha}\right) / 2\right] \cos \left[\left(\phi_{j}-\phi_{\alpha}\right) / 2\right](\alpha=x, y, z$ labels the (ij) bond direction, $\phi_{x, y}=\mp 2 \pi / 3$ and $\phi_{z}=0$ ). In the orbital disordered case this amplitude fluctuated from bond to bond and the macroscopic transport are isotropic. When orbitals are ordered, however, since orbital ordered state is always accompanied by a global anistropy [21], there is one direction along which the hopping amplitude is much smaller than that along the other direction(s), for example, in the FM orbital ordered case $\phi_{i} \equiv \phi$, one can show that whatever the magnitude of $\phi$ is, there is always one direction along which the hopping integral is smaller than one quarter of that along another direction. In the special case of $\phi=\pi$ (corresponding to orbital $d_{x^{2}-y^{2}}$ ), along the $\mathrm{z}$ direction the hopping even vanishes. It is the weaker propagation along this direction that enhances the localization effect of the whole system.

\section{Temperature variation}

Finally let us briefly see the temperature induced MIT. In a given system the electronlattice coupling $g$ and potential fluctuation $W$ are fixed while the spin disorder is temperature 
dependent. For our purpose the temperature dependence of the localized spin orientations is needed. Here we use the mean-field distribution of the orientations, which satisfies the selfconsistent equation $f\left(\theta_{i}, T\right)=F \exp \left(3\left\langle\cos \theta_{i}\right\rangle \cos \theta_{i} T_{c} / T\right)$. In Fig.4 we show the localization length as a function of the temperature. For fixed parameters $g=1.7$ and $W=4 t$, it is found that the MIT temperature $T_{M I} \approx 0.9 T_{c}$ is close to, but not exactly at the Curie temperature $T_{c}$. Note that here the judgement of $\mathrm{M}$ or I phase is whether electrons are localized, different from that in some other references where it is based on the sign of $d \rho / d T$ so that the MIT temperature is always at the resistivity peak. Above $T_{M I}$ and below $T_{c}$, the localization length decreases rapidly with the increase of the temperature due to the sharp drop of the magnetization, as seen from Fig. 3, so that the resistivity can still increase in this temperature region where transport is dominated by variable-range hopping of electrons $[5]$.

\section{SUMMARY}

In summary, by using numerical scaling calculations for the localization effect in the orbital degenerated manganese system, we have shown that within reasonable range of parameters the MIT in manganites can be driven by the joint effect of the DE spin disorder, the on-site electronic potential fluctuation, and the JT distortion. We argue that all these three interactions are relevant to the FM metallic to PM insulate transition in colossal magnetoresistive manganites.

\section{ACKNOWLEDGMENTS}

This work was supported by the Texas Center for Superconductivity at the University of Houston and the Robert A. Welch Foundation, and was also supported in part by the Ames Laboratory, which is operated by Iowa State University for the U.S. Department of Energy (DOE) under Contract No. W-7405-ENG-82. 


\section{REFERENCES}

[1] R. M. Kusters, J. Singleton, D. A. Keen, R. Mcgreevy, and W. Hayes Physica (Amsterdam) 155B, 362(1989); R. von Helmolt, J. Wecker, B. Holzapfel, L. Schultz, and K. Samwer, Phys. Rev. Lett. 71, 2331 (1993).

[2] S. Jin, T. H. Tiefel, M. Mccormack, R. A. Fastnacht, R. Ramesh, and L. H. Chen, Science 264, 413 (1994); P. Schiffer, A. P. Ramirez, W. Bao, and S-W. Cheong, Phys. Rev. Lett. 75, 3336 (1995).

[3] A. Asamitsu, Y. Moritomo, T. Arima, and Y. Tokura, Nature (London) 373, 407 (1995).

[4] J. M. D. Coey, M. Viret, and L. Ranno, Phys. Rev. Lett. 75, 3910 (1995).

[5] C. M. Varma, Phys. Rev. B 54, 7328 (1996).

[6] C. Zener, Phys. Rev. 82, 403 (1951); P. W. Anderson and H. Hasegawa, ibid. 100, 675 (1955); P. -G. de Gennes, ibid. 118, 141 (1960).

[7] J. M. D. Coey, M. Viret, and S. von Molnar, Adv. Phys. 48, 167 (1999); Y. Tokura, and Y. Tomioka, J. Magn. Magn. Mater. 200, 1 (1999).

[8] L. Sheng, D. Y. Xing, D. N. Sheng, and C. S. Ting, Phys. Rev. Lett. 79, 1710 (1997); Phys. Rev. B 56, R7053 (1997).

[9] Q. Li, J. Zang, A. R. Bishop, and C. M. Soukoulis, Phys. Rev. B 56, 4541 (1997).

[10] M. J. Calderón, J. A. Vergés and L. Brey, Phys. Rev. B 59, 4170 (1999).

[11] T. Arima, Y. Tokura, and J. B. Torrance, Phys. Rev. B 48, 17006 (1993); T. Saitoh et al., Phys. Rev. B 51, 13942 (1995).

[12] A. J. Millis et al., Phys. Rev. Lett. 77, 175 (1996); Phys. Rev. B 54, 5405 (1996).

[13] S. Yunoki, J. Hu, A. L. Malvezzi, A. Moreo, N. Furukawa, and E. Dagotto Phys. Rev. Lett. 80, 845 (1998); S. Yunoki, A. Moreo, and E. Dagotto, ibid. 81, 5612 (1998); E. 
Dagotto, T. Hotta, and A. Moreo, Phys. Rep. 344, 1 (2001).

[14] D. P. Arovas and F. Guinea, Phys. Rev. B 58, 9150 (1998).

[15] J. Burgy, M. Mayr, V. Martin-Mayor, A. Moreo, and E. Dagotto Phys. Rev. Lett. 87, $277202(2001)$.

[16] J. A. Vergés, V. Martín-Mayor, and L. Brey, Phys. Rev. Lett. 88, 136401 (2002).

[17] J. C. Slater and G. F. Koster, Phys. Rev. 94, 564 (1955).

[18] J. van den Brink and D. Khomskii, Phys. Rev. Lett. 82, 1016 (1999).

[19] A. MacKinnon and B. Kramer, Z. Phys. B-Condens Matter 53, 1 (1983).

[20] Y. Endoh et al., Phys. Rev. Lett. 82, 4328 (1999).

[21] R. Y. Gu, S. Q. Shen, Z. D. Wang and D. Y. Xing, J. Appl. Phys. 88, 5300 (2000). 


\section{FIGURES}

FIG. 1. Rescaled localization length as a function of the energy $E$. The inset is the scaling parameter $\xi$ as a function of $E$. The length of the bar is taken to be $L=10^{5}$.

FIG. 2. Phase diagram in the PM and FM magnetic background at $x=0.2 . L_{\mathrm{PM}}$ and $L_{\mathrm{FM}}$

are MIT lines in the PM and FM states, respectively. Regions below $L_{\mathrm{PM}}$ and above $L_{\mathrm{FM}}$ are metal and insulator in both the PM and FM cases, while the region in between is insulator in the PM state and metal in the FM state.

FIG. 3. Comparison of the localization lengths in the orbital disordered (open symbols) and C-type AF ordered (solid symbols) configurations in the PM state at $g=1.4$ and $W=4 t$.

FIG. 4. Temperature dependence of the localization lengths at $g=1.7$ and $W=4 t$. 


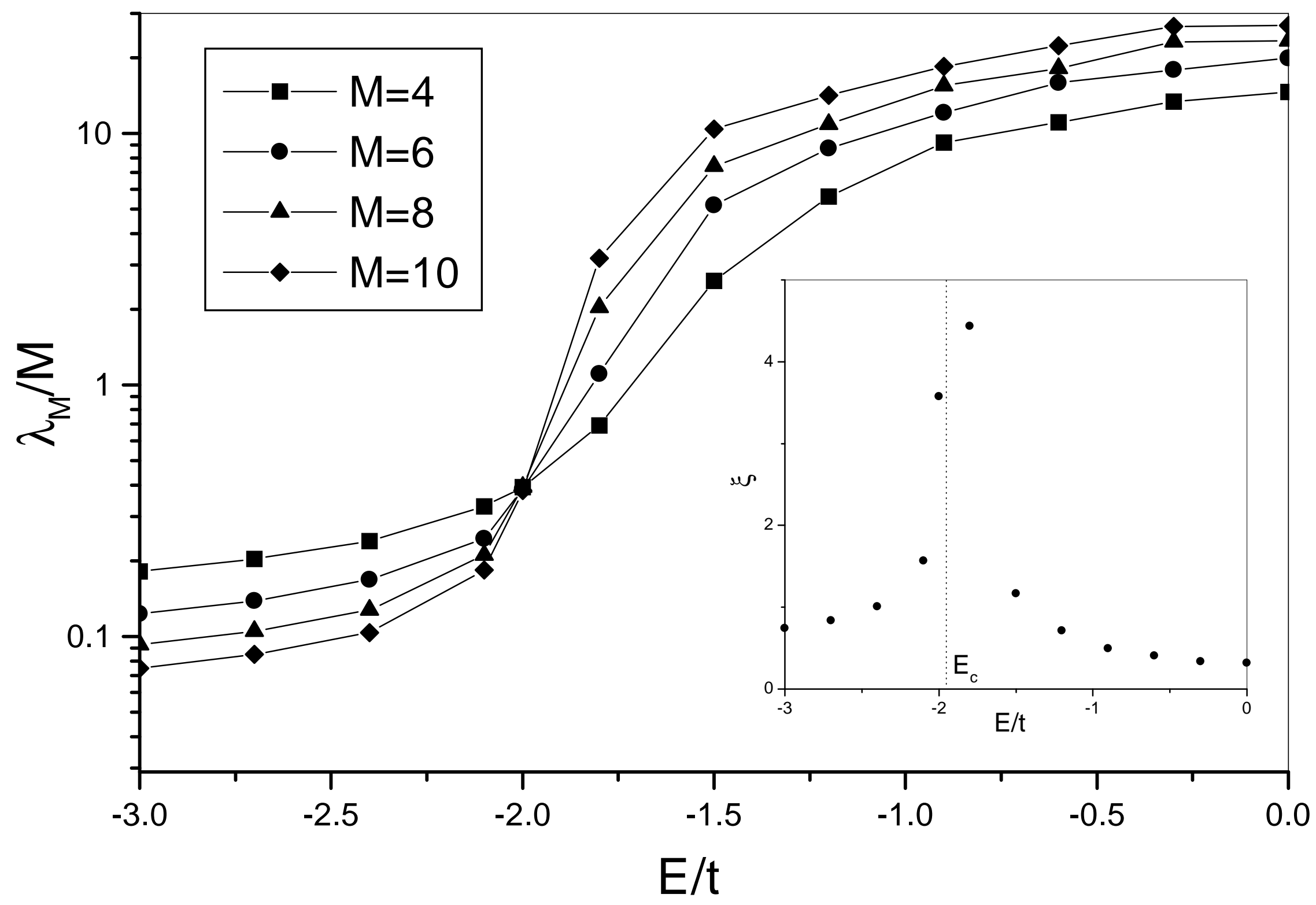




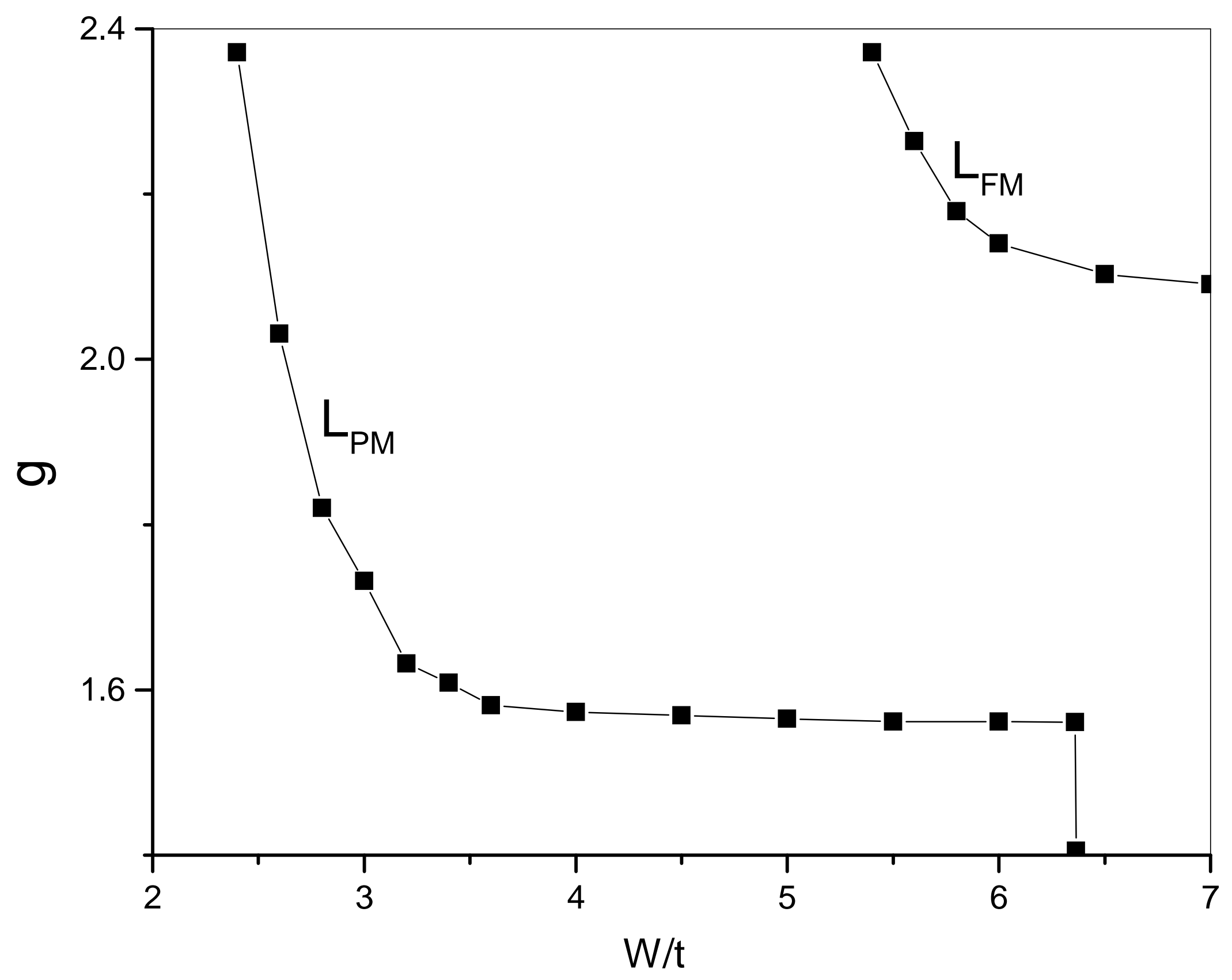




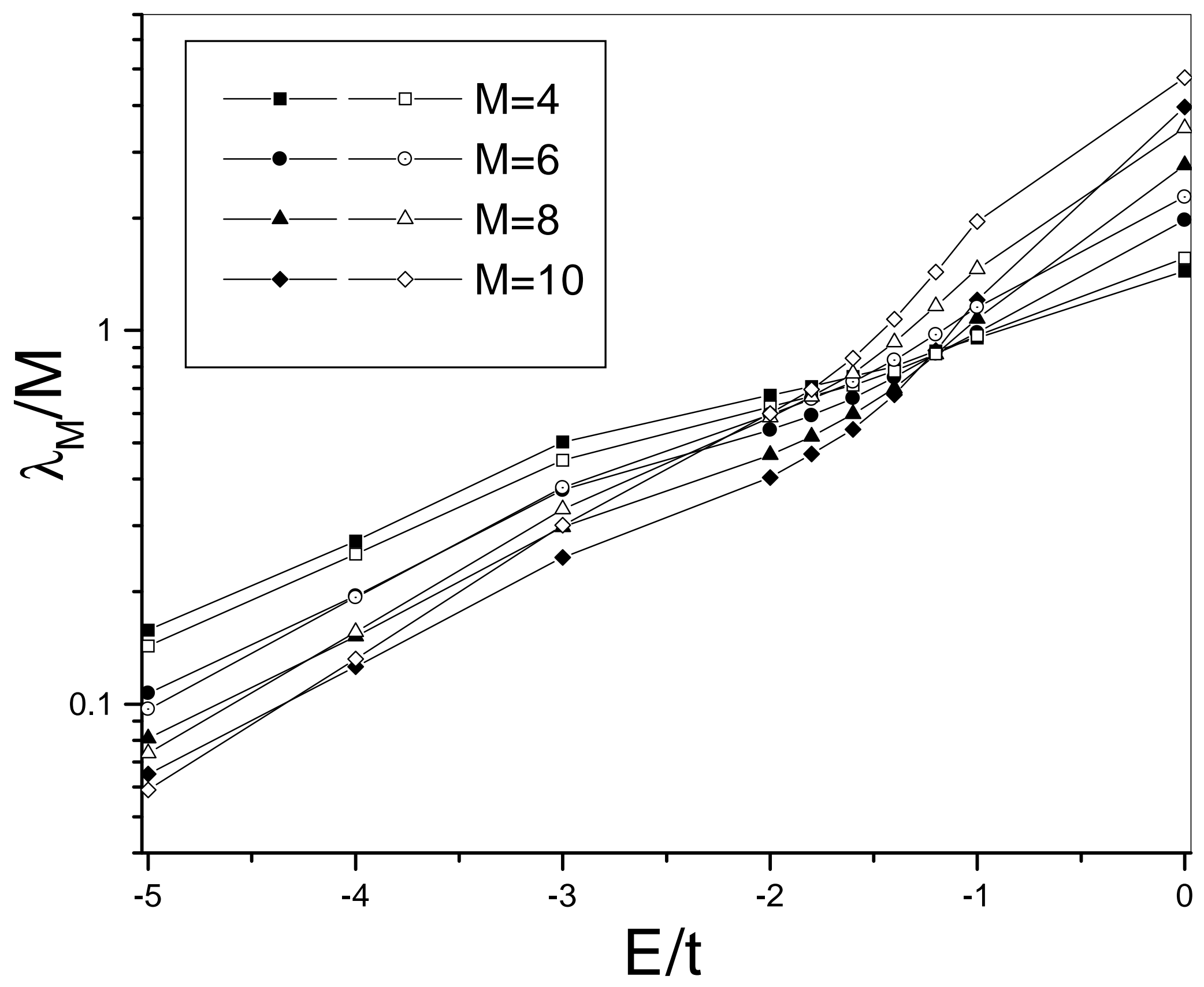




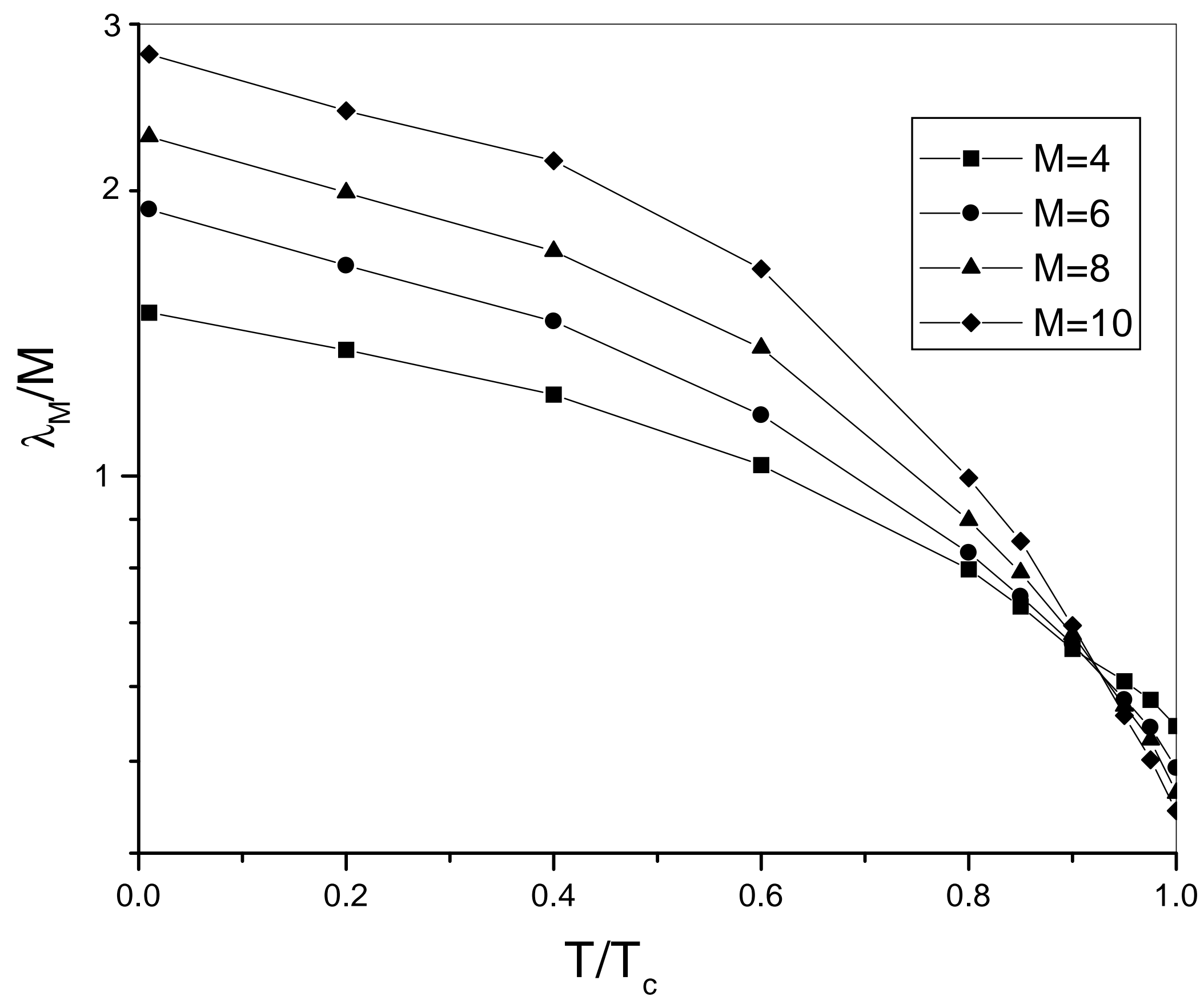

